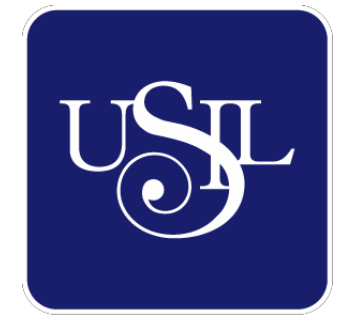

UNIVERSIDAD

SAN IGNACIO

DE LOYOLA

FACULTAD DE HUMANIDADES

Carrera de Psicología

\title{
BIENESTAR PSICOLÓGICO Y CALIDAD DE VIDA PROFESIONAL EN MIEMBROS DE LA POLICÍA NACIONAL DEL PERÚ EN PANDEMIA POR COVID- 19
}

Tesis para optar el Título Profesional de Licenciado en Psicología

\section{MARIELLA JESÚS TRUJILLO NAUPARI \\ (0000-0002-7696-3722)}

Asesor:

Mg. Carmen Magali Meléndez Jara

(0000-0003-1446-4837)

Lima - Perú

2020 


\section{Agradecimiento}

Por el apoyo que brindaron para que la presente investigación sea posible, se les agradece:

A Dios por la fortaleza, y la capacidad para afrontar cada dificultad que se atraviesa y porque creo que sus planes son perfectos.

A mi asesora Mg. Carmen Magali Meléndez Jara, quien me apoyo con su compromiso, perseverancia y sus conocimientos en cada etapa del desarrollo de la presente investigación para cumplir con los objetivos planteados.

A cada uno de los miembros de la Policía Nacional del Perú, por el interés de compartir sus experiencias durante la pandemia por la COVID-19 y su participación voluntaria que permitió llevar a cabo el proyecto.

Finalmente, agradezco a mi familia y amigos por cada palabra de aliento que permitieron motivarme y renovar energías que me animan a crecer como persona y como profesional.

Gracias a todos. 


\section{Resumen}

El presente estudio tuvo como objetivo analizar la relación entre el bienestar psicológico y calidad de vida profesional en miembros de la Policía Nacional del Perú en pandemia por COVID-19. Para ello se contó con la participación de 133 miembros de la PNP entre los 20 y 30 años, que laboraban en distintos distritos de Lima Metropolitana, de los cuales 7 (7.52\%) fueron mujeres y $123(92.48 \%)$ fueron varones. Siguiendo un diseño de investigación de tipo correlacional-simple, y adicionalmente se analizó comparativamente a los participantes con y sin diagnóstico de COVID-19, asimismo el muestreo utilizado fue el no probabilístico por conveniencia. Se utilizó la Escala Bienestar Psicológico para adultos (EBP-A), diseñada por Casullo (2002) y validada en Perú por Domínguez (2014), y la Escala de Calidad de Vida Profesional (CVP-35) elaborada por Cabezas (1998) y adaptada en Perú por Grimaldo y Reyes (2013). En cuanto, a los análisis estadístico se hizo uso de la $\mathrm{R}$ de Pearson y de la T de Student, evidenciando que no existe una correlación significativa entre las variables en cuestión, con respecto al análisis comparativo se encontró que la ausencia de asociación entre las variables de estudio y los grupos; sin embargo, se tomó en cuenta el insignificante tamaño del defecto para llevar a cabo las conclusiones de la investigación.

Palabras clave: Bienestar psicológico, calidad de vida profesional, pandemia. 


\begin{abstract}
The objective of this study was to analyze the relationship between Psychological well-being and the Quality of professional life in members of the National Police of Peru in the COVID19 pandemic. The sample was of 133 members of the PNP between 20 and 30 years of age, they work in different districts of Metropolitan Lima, made up of 7(7.52\%) were women and $123(92.48 \%)$ were men. The study follows a simple and non-experimental simplecorrelational research design, additionally development a comparative analysis respect to the members with and without a COVID-19 diagnosis, and the sample was chosen by a nonprobability sampling. This study was used the Psyhological Well-being Sale for adults (EBPA), made by Casullo (2002) and valid in Peru by Dominguez (2014), and the Professional Quality of Life Scale (PQL-35) design by Cabezas (1998) and adapted in Peru by Grimaldo and Reyes (2013). In this study was used the R of Pearson and T student. The results showed that there is no significant correlation between the study variables, and respect to the comparative analysis it was found that the absence of association between the variables of study and the groups; is very important to mention that the insignificant size of the defect was taken into account in drawing the conclusions of the investigation.
\end{abstract}

Key words: Psychological well-being, quality of professional life, pandemic. 
Introducción........................................................ 1

Objetivos de la investigación....................................... 12

Objetivo general....................................... 12

Objetivo específico ...................................... 12

Hipótesis ............................................... 13

Hipótesis especificas................................... 13

Método................................................................. 15

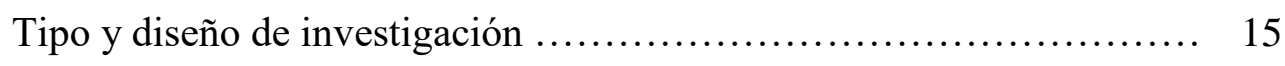

Participantes................................................ 15

Criterios de inclusión $\ldots \ldots \ldots \ldots \ldots \ldots \ldots \ldots \ldots \ldots \ldots \ldots \ldots \ldots \ldots \ldots$

Criterios de exclusión ...................................... 16

Instrumentos de recolección de información...................... 16

Procedimiento................................................ 18

Análisis de datos..................................................... 19

Resultados ............................................... 20

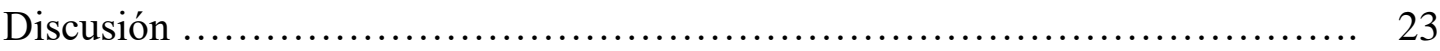

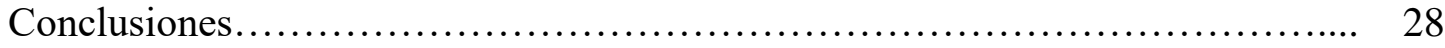

Referencias ....................................................... 32

Apéndices......................................................... 40

Apéndice A: Consentimiento informado ........................ 40

Apéndice B: información personal.................................. 41

Apéndice C: Escala de BIEPS-A ................................ 42

Apéndice D: Escala de CVP-35 .................................. 43 


\section{Índice de tablas}

Tabla 1: Características Sociodemográficas de los participantes...................... 16

Tabla 2: Análisis descriptivo de las dimensiones de Bienestar psicológicas Calidad de vida profesional............................................. 20

Tabla 3: Análisis correlacional entre las dimensiones de Bienestar psicológico y Calidad de vida profesional............................................ 21

Tabla 4: Comparación del Bienestar Psicológico y Calidad de Vida Profesional conforme al diagnóstico COVID-19...................................... 


\section{Introducción}

Actualmente a nivel mundial se atraviesa una de las mayores de crisis sanitarias debido a la enfermedad producida por el nuevo Coronavirus Disease (COVID-19), reportada por primera vez en Wuhan (China) el 31 de diciembre de 2019; la llegada de dicha enfermedad al Perú en marzo del pres

ente año ha representado no solo el peligro físico a la exposición del virus si no también ha ocasionado impactos negativo en la salud mental, tal como lo indican los profesionales del Colegio de Sociólogos del Perú (2020) y el Ministerio de Salud del Perú (2020), quienes reafirman que el estrés, los problemas psicosociales y la depresión son algunos de los síntomas que presenta la población en general, apoyando a los resultados de un estudio realizado en la población general de China, donde obtuvieron que el $53.8 \%$ presenta un nivel moderado a severo con respecto al impacto psicológico, un $16.5 \%$ presentaron síntomas depresivos moderados a severos, $28.8 \%$ reporto síntomas de ansiedad moderada a severa y $8.1 \%$ reporto niveles de estrés moderados a severos (Vargas, 2020).

Es así que se observa una afectación negativa con respecto al Bienestar Psicológico en la población en general y en especial en aquellos profesionales que se encuentran en la primera línea de lucha frente al COVID-19 entre ellos tenemos a los miembros de la Policía Nacional del Perú (PNP), quienes hasta agosto del 2020 sufrieron 379 muertes y más de 23 mil contagios por COVID-19 entre sus 130 mil efectivos desde que el gobierno decreto el estado de emergencia nacional por pandemia, además unos 4 mil miembros de la PNP cumplieron cuarentena obligatoria hasta agosto del presente año, por ser parte de la población vulnerable ya sea por edad o por presentar enfermedades coexistentes (El Peruano, 2020), a pesar de estas cifras alarmantes no existen estudios nacionales con respecto al impacto de la pandemia en la salud mental de dicha población. . 
El riesgo de contagio es alto en estos servidores públicos, debido a que llevan a cabo funciones de velar por la seguridad y orden, cumplimiento de las distintas medidas adoptadas por el Gobierno Peruano desde que se inició el estado de emergencia, como: el cierre de fronteras, aislamiento domiciliario, toque de queda, entre otros (Decreto Supremo $\mathrm{N}^{\circ}$ 044, 2020; Decreto Supremo $N^{\circ}$ 094, 2020; Decreto Supremo $N^{\circ} 156,2020$ ); dichas medidas adoptadas han generado mayor demanda laboral en los efectivos de la PNP quienes han sufrido cambios en su horario de trabajo, pasando de un horario de $24 \times 24$ a un horario de 48x48 (Decreto de Urgencia $N^{\circ} 012,2020$ ). Dichas demandas generan implicancias negativas físicas y psicológicas tales como, una elevada exigencia con respecto a su estado de alerta y en algunos casos a experimentar duelo debido a la pérdida de sus colegas y/o familiares, asimismo estos pueden ser factores desencadenantes de estrés, depresión, ansiedad y temor, impactando de esa manera en la calidad de vida profesional y por ende en su bienestar psicológico (Caycho, Carbajal, Vilca, Heredia \& Gallegos, 2020).

Es importante estudiar ambas variables, ya que por un lado el bienestar psicológico permite que las personas desarrollen y practiquen adecuadamente sus habilidades individuales (Mayordomo, Sales, Satorres \& Meléndez, 2016), mientras que una adecuada calidad de vida profesional influirá positivamente en la percepción de la persona con respecto a varias áreas de su vida como: la personal, profesional y social permitiendo de esta manera que esta mantenga una adecuada salud mental y estabilidad emocional (Moya, Caro \& Monsalves, 2017).

Las actuales actividades realizadas por los miembros de la PNP y las consecuencias negativas en su salud físicas y psicológicas ocasionadas por la actual situación de pandemia conllevan al planteamiento del siguiente problema de investigación: ¿Existe relación significativa y positiva entre el Bienestar Psicológico y la Calidad de vida profesional en situación de pandemia por COVID-19 en los miembros de la PNP de Lima Metropolitana? 
Por otro lado, se necesitó conocer si existían diferencias entre el personal policial con y sin diagnóstico de COVID-19 en las variables sujetas a estudio, contando con un antecedente en esta misma línea de Balluerka et al. (2020) quienes reportaron consecuencias psicológicas del COVID-19 y el confinamiento en una población española adulta, indicando un incremento porcentual de los sentimientos de culpa, irritabilidad, baja motivación y pobre autoconfianza entre personas que han tenido síntomas y/o han sido diagnosticados con COVID-19, y/o se mantienen en cuarentena por la enfermedad a diferencia de las que no han presentado síntomas y/o no han sido diagnosticados. Estos resultados nos llevan a plantearnos la necesidad de determinar si existen diferencias en el bienestar psicológico y la calidad de vida profesional entre los miembros de la PNP con y sin diagnóstico positivo de COVID-19.

\section{Bienestar psicológico}

Se entiende por bienestar psicológico al correcto funcionamiento del conjunto de capacidades, crecimiento personal y el sentirse bien con uno mismo permitiendo de esa manera un equilibrio emocional (Torres, Olivella, Cudris-Torres, Bahamón, \& Gil; 2020). Los estudios relacionados con esta variable han estado influidos por dos posturas: hedónica (centrado en la búsqueda del placer) y eudaimónica (Ryan \& Deci, 2001). La presente investigación se basará en la postura eudaimónica, la cual hace referencia al crecimiento personal, al desarrollo de las propias capacidades y al deseo de la autorrealización personal a través del alcance de las metas (Muratori, Zubieta, Ubillos, Gonzáles \& Bobowik, 2015; Ryan \& Deci, 2001).

Ryff (1989) propone un modelo multidimensional con el objetivo de comprender mejor el bienestar psicológico el cual es dividido en seis dimensiones: (1) Auto-aceptación, se refiere a la aceptación de las experiencias pasadas y aceptar los distintos aspectos que conforman la vida presente de la persona, independientemente si son aspectos positivos o 
negativos; (2) Capacidad de mantener relaciones positivas/vínculos sociales, implica lograr mantener relaciones interpersonales saludables, es decir, desarrollar confianza, empatía y comprensión con los otros; (3) Autonomía, involucra la autoevaluación de las propias capacidades para luchar contra las presiones sociales, y generar autodeterminación e independencia; (4) Dominio del entorno/control de situaciones, tiene que ver la habilidad de control de los factores externos con el fin de construir un ambiente adecuado para el desarrollo y satisfacción personal; (5) Crecimiento personal, implica el desarrollo individual y del propio potencial para hacer uso de las distintas capacidades; Y finalmente (6) Propósito de vida/Proyecto, indica la definición de metas y deseos de cumplirlas que dan sentido a las experiencias de la persona (Pineda, Castro \& Chaparro, 2018; Ryff \& Keyes, 1995).

Por otro lado, la postura eudaimónica presenta asociaciones con la salud física y mental, referente a los factores biológicos y salud física estos son más consistente con respecto a la postura hedónica (Vázquez \& Castilla, 2007), asimismo, se hallaron que tiene relación con bajos niveles de riesgo cardiovascular, altos niveles del colesterol de tipo HDL (colesterol bueno), bajo peso corporal y una adecuada regulación endocrina evidenciando bajos niveles de cortisol en la saliva (Friedman, Hayney, Gayle, Burton \& Ryff, 2007). Por otro lado, la postura eudaimónica y hedónica están relacionado con la activación de la corteza pre-frontal, en especial con el lado izquierdo (Urry et al., 2004). Además en lo referente a su asociación con la salud psicológica, la postura eudaimónica reduce el riesgo del desarrollo de las enfermedades mentales (Mjosund et al., 2015), asimismo, está relacionado con altos niveles de felicidad, auto-aceptación, adecuadas relaciones interpersonales, autonomía, desarrollo de las capacidades para afrontar problemas y con un nivel adecuado de autoestima (Hun, Urzúa, López, Escobar \& Leiva, 2019; Mesa, Pérez, Nunes \& Menéndez, 2019; Muratori et al., 2015; Uribe, Ramos, Villamil \& Palacio, 2018).

Por otro lado, en cuanto a los factores de influencia García, Hernández, Espinosa y 
Soler (2020) mencionan para que exista un adecuado desarrollo del bienestar psicológico influyen muchas variables a nivel personal y social, entre ellas el autocontrol, autoconocimiento y autoaceptación (Liberal, Escudero, Cantallops \& Ponseti, 2014), y las adecuadas condiciones ambientales, como la relaciones familiares positivas y el sentirse apoyado por sus relaciones interpersonales (Ballesteros, Medina \& Caycedo, 2006).

En tal sentido, se debe de tener en cuenta que los miembros de la PNP, como profesionales que actúan en primera línea frente a la lucha contra el COVID-19, están expuestos a condiciones ambientales no adecuadas y que no favorecen el bienestar psicológico, por lo que deben de elaborar estrategias para que les permitan mantenerse emocionalmente estables para poder cumplir con su labor.

Con respecto a las investigaciones en este campo teórico, tenemos que, Muratori et al., (2015), realizaron un estudio considerando como objetivo el comparar en estudiantes universitarios españoles y argentinos, los niveles de felicidad y el bienestar psicológico, participaron 193 argentinos (54 hombres y 193 mujeres) y 162 españoles (45 hombres y 117 mujeres) entre los 18 y 64 años; para la recolección de datos se hizo uso de la Escala de Felicidad Subjetiva (Lepper, 1999) y la Escala de Bienestar Psicológico de Ryff (Diaz et al. 2006). Dicho estudio obtuvo como resultado que la muestra española obtuvo más niveles de felicidad, mientras que la muestra argentina presentó mayor bienestar psicológico. Asimismo, las mujeres argentinas perciben mayor dominio del entorno que los hombres, y los españoles mantienen una mayor frecuencia en sus adecuadas relaciones interpersonales. Llegando a la conclusión que la felicidad actuaba como un mediador para el desarrollo del bienestar psicológico.

Por otro lado, García, Manquián y Rivas (2016) realizaron una correlación entre el bienestar psicológico, las estrategias de afrontamiento y el apoyo social en cuidadores informales en Chile, los participantes fueron 63 hombres y 131 mujeres, siendo un total de 
194 personas entre los 18 y 78 años. Para la recolección de datos hicieron uso del Inventario del Brief COPE (Carver, 1997), el cual evalúa las estrategias de afrontamiento, la Flourishing Scale de Diener (Dienes et al., 2009) para la evaluación del bienestar psicológico y la Escala Multidimensional de Apoyo Social Percibido (MSPSS) de Zimer, Dahlem, Zimer y Farley (1988). Luego del análisis de los datos recolectados obtuvieron, que sí existe una correlación significativa entre las estrategias de planificación, aceptación y apoyo social percibido con el bienestar psicológico, siendo el apoyo de las amistades el principal predictor del bienestar psicológico.

Asimismo, Rangel, Mayorga, Peinado y Barrón (2017) realizaron un estudio con el objetivo de evaluar el efecto de la práctica regular de la actividad y el autoconcepto físicos ante la percepción del bienestar psicológico. La muestra estuvo conformada por 532 estudiantes universitarias mexicanas entres los 18 y 26 años. Para la realizar la recolección de datos utilizaron: la International Physical Activity Questionnaires (IPAQ) de Craig et al., (2003), el Cuestionario de autoconcepto físico modificado (CAF-M) de Goñi, Ruiz de Azúa y Rodriguez (2006) y la Escala de bienestar psicológico (EBP) de Díaz et al., (2006). Dicha investigación tuvo como resultado que la constante práctica de la actividad física a través del autoconcepto físico tiene un efecto indirecto en la percepción del bienestar psicológico, asimismo no se encontró un efecto directo de entre la competencia motora y la autoaceptación. En conclusión, se puede observar que la práctica constante de la actividad física influye directamente de forma significativa en el atractivo físico, la autoaceptación y el crecimiento personal.

En esta misma línea, Hun et al., (2019) desarrollaron un estudio con la finalidad de analizar la relación entre las características del comportamiento alimentario y el bienestar psicológico en los estudiantes universitarios del norte de Chile. La muestra de estudio estuvo conformada por 647 alumnos, de los cuales 498 fueron mujeres y 149 varones entre los 18 y 
35 años; para la recolección de los datos hicieron uso del cuestionario de bienestar psicológico de Ryff (1989) y el cuestionario holandés de conductas alimentarias (Ducht Eating Behavior Questionnaire-DEBQ). Y obtuvieron como resultado la relación significativa entre las variables estudiadas, indicando que la ingesta emocional (hipótesis que sostiene que las emociones y el estado de ánimo influyen en la conducta alimentaria) tiene un mayor impacto en el bienestar psicológico, mientras la ingesta externa (hace inferencia a los factores externos, como el sabor, textura, olor o color de los alimentos) no presenta algún efecto sobre el bienestar psicológico.

A nivel nacional se encontró que Matalinares, Díaz, Raymundo, Baca, Uceda \& Yaringaño (2016) desarrollaron un estudio con el objetivo que correlacionar los métodos de afrontamiento del estrés y del bienestar psicológico en una muestra peruana de 934 estudiantes universitarios, de los cuales 534 fueron de la ciudad de Lima y 400 de Huancayo, entre los 16 y 25 años, siendo 390 varones y 544 mujeres. Hicieron uso de la Escala de Modos de Afrontamiento al Estrés (COPE) elaborada por Carver, Sheir y Weintraub (1989) y la Escala de Bienestar Psicológico de Ryff (1989). Luego de los análisis correspondientes obtuvieron como resultados que existe una relación significativamente directa entre el modo de afrontamiento del estrés y el bienestar psicológico, además con respecto al modo de afrontamiento centrado en el emoción hallaron que los estudiantes de Huancayo tienden a modificar cualquier situación adversa o estresante que se les presente mediante una perspectiva más positiva de la situación; mientras que por otro lado los estudiantes de la ciudad de Lima presentaron mayor puntuación con respeto a la necesidad de crecimiento personal, desarrollo de sus capacidades y habilidades, mantenimiento de la dependencia y autonomía personal.

Por su parte, Tello (2018), llevó a cabo una investigación con el objetivo de evaluar la relación entre el bienestar psicológico y calidad de vida en una muestra de 260 universitarios 
de la carrera de psicología de Lima, de los cuales 76 fueron varones y 184 mujeres. Para recolectar los datos hizo uso de la Escala de bienestar Psicológico de Ryff (1989) y la Escala de Calidad de Vida de Bernes y Olson (1982). Con respecto a los resultados obtuvo que existe una relación directamente proporcional entre bienestar psicológico y calidad de vida profesional, asimismo obtuvo que los participantes presentaban un nivel promedio de bienestar psicológico

Chávez (2015), realizo un estudio con la finalidad de analizar la relación entre el bienestar psicológico y la calidad de vida en personas con discapacidad motriz de centros de integración de discapacitados de la ciudad de Trujillo, siendo un total de 134 participantes entre los 25 y 60 años. Para el desarrollo de la investigación utilizó la escala de bienestar psicológico de Sánchez (1998) y la escala de calidad de vida de Olson y Barnes (1982). Con respecto a los resultados hallo una correlación altamente significativa entre el bienestar psicológico y calidad de vida profesional.

\section{Calidad de vida profesional}

En cuanto a la Calidad de vida profesional, Cabezas (1998) la define como la satisfacción percibida por el profesional frente a las demandas de su labor, es decir, que consiste en la percepción del individuo entre las exigencias laborales y los recursos personales con los que dispone para afrontarlos.

Existen diferente factores que influyen en la calidad de vida profesional: (1) Cargas o demandas en el trabajo, se refiere a la cantidad de trabajo, demandas físicas y psicológicas que percibe el trabajador; (2) Motivación intrínseca, hace inferencia a la motivación personal, guiadas por las necesidades individuales de cada persona que guían las conductas hacia la satisfacción del desarrollo profesional; (3) Apoyo directo, involucra al apoyo externo que percibe la persona por parte de sus supervisores o jefes inmediatos y compañeros de trabajo, 
ligado al reconocimiento de su desempeño laboral (Grimaldo, 2010; Zuazo, 2018).

Los factores que influyen en la calidad de vida profesional puedes ser de carácter laboral, como: el ambiente físico donde se desarrolla la actividad laboral, haciendo referencia a la iluminación, ventilación, comodidad y a los riesgos físicos, químicos y biológicos que se presente dentro del ambiente; los accidentes laborales; ambiente tecnológico, la falta de instrumentos o herramientas tecnológicas pueden causar incomodidad en el trabajador; el salario, es uno de los conceptos más valorados, ya que funciona como un estímulo de motivación extrínseca; la estabilidad en el puesto de trabajo y la posibilidad de ser promovido, es un factor importante ya que está relacionado con el compromiso y motivación; el horario de trabajo, los trabajadores que mantienen largos turnos y horarios rotativos de trabajo presentan problemas con respecto a sus relaciones interpersonales y familiares, así como problemas al dormir (insomnio) y cansancio físico y mental; y la sobrecarga laboral, existen dos tipos de demandas: las relacionadas con el aumento de horas de trabajo y de las funciones (cuantitativas) y las referentes a la demanda de una mayor responsabilidad, competencia y habilidades (cualitativas). Asimismo, influyen factores de carácter personal, como: características de la personalidad, edad, sexo, apoyo familiar, estado civil, etc. (Moya, Caro \& Monsalves, 2017).

La calidad de vida profesional puede verse afectada por la exposición constante al estrés, debido a que representa una carga emocional y puede deteriorar la calidad de vida y la capacidad de realizar adecuadamente su labor (Somoray, Shakespeare, Finch \& Armstrong, 2017), siendo esta una posible actual situación de los miembros de la PNP quienes actualmente se encuentran bajo mucha demanda laboral debido a la pandemia sanitaria por el COVID-19 pudiendo ocasionar efectos negativos con respecto a su bienestar psicológico.

Por otro lado, en relación con las investigaciones sobre este campo teórico de estudio de esta variable, se encuentra que, Salgado y Leria (2017), realizaron una investigación con el 
objetivo de analizar los niveles del síndrome de quemarse en el trabajo (Burnout) y la percepción de calidad de vida profesional en función del estilos de personalidad de Torgensen, para ello participaron un 212 profesores de educación primaria de Chile. Hicieron uso del Cuestionario de Vida Profesional (CVP-35), el Cuestionario de estar quemado por el trabajo (CESQT) y el Inventario de los Cinco Factores (NEO-FFI). Encontraron como resultados que los hombres tenían mayor puntuación con respecto a la escala de indolencia y culpa; mientras que las mujeres presentan una mayor puntuación en la ilusión por el trabajo. Concluyeron que el estilo de personalidad está relacionado con la calidad de vida profesional y el síndrome de Burnout.

Del mismo modo, Moya, Caro y Monsalves (2017), desarrollaron un estudio con la finalidad de conocer la percepción de la Calidad de Vida Profesional (CVP) de los docentes de Odontología de una institución de educación superior; los participantes fueron 102 docentes de Odontología de un centro de educación superior de la región metropolitana de Chile. Para la recolección de los datos hizo uso del cuestionario de Calidad de Vida Profesional (CVP-35). Obtuvieron como resultados que los participantes presentaban un nivel medio-alto con respecto a la calidad de vida profesional, tomando mayor importancia a la motivación intrínseca.

Uribe, Ramos, Villamil y Palacio (2018), realizaron un estudio con el objetivo de determinar si existía una relación entre la calidad de vida profesional y el Síndrome de Burnout en 200 efectivos de la Policía Nacional del Perú (PNP) de Lima Metropolitana entre los 19 y 60 años, de los cuales 55 eran mujeres y 145 varones. Con respecto a la recolección de datos hizo uso del Inventario de Burnout de Maslach (Maslach \& Jackson, 1981), dicho cuestionario evalúa el Agotamiento emocional, despersonalización y realización personal, también utilizó el Inventario de Calidad de Vida Profesional (CVP) (Cabezas, 1998), el cual evalúa cargas de trabajo, motivación intrínseca y el apoyo directo. Se obtuvo como resultado 
una correlación negativa y significativa entre la dimensión distanciamiento emocional con el apoyo directivo y la motivación intrínseca; por el contrario, obtuvo una relación positiva y significativa con la carga de trabajo. Adicionalmente, se encontró una correlación positiva y significativa entre la dimensión realización personal con el apoyo directivo y motivación intrínseca; y de forma opuesta una correlación negativa y significativa con la carga de trabajo. El estudio llegó a la conclusión que existe relación entre el Síndrome de Burnout y la calidad de vida profesional en miembros de la PNP de Lima Metropolitana, reafirmando la importancia de fortalecer la correcta función de dichas instituciones.

\section{Justificación}

De acuerdo a las investigaciones referidas, respecto a las variables de bienestar psicológico y calidad de vida profesional en contexto de pandemia por Covid-19, se encontró vacíos teóricos observados en la literatura consultada, tanto a nivel nacional e internacional no se hallaron estudios que relacionen ambas variables por lo que se pretende cubrir dicho vacío en el campo de la investigación en el Perú y de esa manera lograr un aporte teórico sobre el tema en mención en una población poco estudiada, siendo esta la de los miembros de la Policía Nacional del Perú (PNP), profesionales que forman parte de una institución del estado que actúa en primera línea de defensa para mantener el orden interno entre los

ciudadanos del estado peruano, lo cual implica que se encuentren con mayor riesgo de contagio del COVID-19 y por ende de presentar implicancias negativas en su salud física y psicológica.

En tal sentido el presente estudio se justifica a nivel teórico debido a que buscó cubrir el vacío antes mencionado, analizando el comportamiento de la variable bienestar psicológico a partir de la postura eudaimónica y del modelo multidimensional planteado por Ryff (1989). Así mismo entender los fenómenos relacionados con la variable calidad de vida profesional, y 
de esta manera reforzar la importancia de realizar investigaciones en base a la evidencia empírica obtenida.

Por otra parte, la investigación se justifica a nivel práctico ya que permitirá elaborar proyectos sociales que brinden herramientas y estrategias a los miembros de la PNP y de esa manera mejorar la calidad de vida de dichos profesionales.

Finalmente, en base a los antecedentes teóricos señalados anteriormente, este estudio se justifica a nivel metodológico debido a que se aplicó herramientas válidas y confiables adaptadas a nuestro contexto nacional, aportando evidencias en las propiedades psicométricas de los instrumentos utilizados. Aunado a ello, al enfocarnos en una población de alto riesgo de contagio permitirá abrir el camino sobre el estudio de fenómenos similares.

\section{Objetivos de la Investigación}

Con respecto a lo detallado se plantea como objetivo general determinar si existe relación significativa y positiva entre el bienestar psicológico y la calidad de vida profesional en pandemia por COVID-19 en miembros de la policía nacional del Perú de Lima Metropolitana. Y con respecto a los objetivos específicos, estos se propusieron en base a las asociaciones del bienestar psicológico y la calidad de vida profesional, establecida en investigaciones que se revisaron como antecedentes, y estás son:

- Determinar si existe una relación significativamente negativa entre el factor de cargas o demandas en el trabajo de la calidad de vida profesional con las dimensiones de bienestar psicológico en la muestra sujeta de estudio.

- Determinar si existe una relación significativamente positiva entre el factor de motivación intrínseca de la calidad de vida profesional con las dimensiones de bienestar psicológico en la muestra sujeta estudio. 
- Determinar si existe una relación significativamente positiva entre el factor de apoyo directo de la calidad de vida profesional con las dimensiones de bienestar psicológico en la muestra sujeta estudio.

Además, se plantearon objetivos en base a una asociación comparativa del bienestar psicológico y la calidad de vida profesional según diagnóstico positivo de COVID-19 en la muestra sujeta a estudio.

- Determinar si existen diferencias en el bienestar psicológico entre los miembros de la PNP con y sin diagnostico positivo de COVID-19.

- Determinar si existen diferencias en la calidad de vida profesional entre los miembros de la PNP con y sin diagnóstico positivo de COVID-19.

\section{Hipótesis}

Con respecto a las hipótesis se plantea que sí existe una relación significativa y positiva entre el bienestar psicológico y la calidad de vida profesional en pandemia por COVID-19 en miembros de la Policía Nacional del Perú de Lima Metropolitana. Asimismo, con respecto a las hipótesis específicas se determinaron las siguientes:

1. Los miembros de la PNP de Lima Metropolitana presentan una relación significativamente negativa entre el factor de cargas o demandas en el trabajo de la calidad de vida profesional con las dimensiones de bienestar psicológico.

2. Los miembros de la PNP de Lima Metropolitana presentan una relación significativamente positiva entre el factor de motivación intrínseca de la calidad de vida profesional con las dimensiones de bienestar psicológico en la muestra sujeta estudio.

3. Los miembros de la PNP de Lima Metropolitana presentan una relación significativamente positiva entre el factor de apoyo directo de la calidad de vida profesional con las dimensiones de bienestar psicológico en la muestra sujeta estudio. 
Adicionalmente, se plantea las siguientes hipótesis:

4. Los miembros que sí han sido diagnosticados positivamente con el COVID-19 presentan menores niveles con respecto al bienestar psicológico.

5. Los miembros que no han sido diagnosticados positivamente con el COVID-19 presentan mayores niveles con respecto a la calidad de vida profesional. 


\section{Método}

Tipo y diseño de investigación

La presente investigación utilizó una estrategia asociativa y transversal. El diseño usado fue correlacional - simple, debido a que tiene como objetivo buscar la relación funcional entre el bienestar psicológico y la calidad de vida profesional en los miembros de la PNP durante la pandemia por COVID-19 en Lima metropolitana. Adicionalmente, se utilizó un tipo de estudio comparativo, con un diseño de corte transversal debido a que busca comparar las variables en cuestión en la muestra sujeta a estudio en un momento temporal determinado (Ato, López \& Benavente, 2013).

\section{Participantes}

En la presente investigación se utilizó el muestreo no probabilístico por conveniencia con consentimiento informado (Hernández, Fernández \& Baptista, 2014; Otzen \& Manterola, 2017). Para calcular el tamaño de la muestra se tomó en cuenta la magnitud del efecto (ME) obtenidos de las investigaciones previas, posteriormente fue analizado en el software G*Power 3.1.7, obteniendo con muestra mínima de 120 participantes.

Se contó con la participación de 133 miembros de la PNP de diferentes distritos de Lima Metropolitana, de entre 20 a 30 años $(M=25.7 ; D E=2.61), 7$ efectivos fueron mujeres (7.52\%) y 123 fueron varones (92.48\%). Todos los miembros de la PNP ejercían actividades tipo operativo, de los cuales 58 fueron diagnóstico con COVID-19 (43.61\%) y 75 no reportaron diagnóstico de COVID-19 (56.39\%), asimismo, 61 (45.86\%) laboraran en distritos de Lima centro, 60 (45.11\%) laboraban en distritos de Lima este, 11 (8.27\%) laboraban en distritos de Lima norte y $1(0.75 \%)$ en distritos de Lima sur. 
Tabla 1

Características Sociodemográficas de los participantes.

$$
\text { Frecuencia Porcentaje }
$$

Horario de trabajo

$\begin{array}{ccc}24 \times 24 & 114 & 85.71 \% \\ 48 \times 48 & 2 & 1.50 \% \\ 8 \text { horas } & 17 & 12.78 \%\end{array}$

Estado civil

$\begin{array}{rcc}\text { Soltero } & 97 & 72.93 \% \\ \text { Conviviente } & 19 & 14.29 \% \\ \text { Casado } & 16 & 12.03 \% \\ \text { Divorciado } & 1 & 0.75 \%\end{array}$

Por otro lado, como criterio de inclusión se consideró a los miembros de la PNP que se vieron expuestos a la vigilancia de la seguridad en las calles, la edad de los participantes oscilo entre los 20 y 30 años de edad, y que laboraban en los distritos de Lima Metropolitana. Como criterio de exclusión, no se tuvo en cuenta a los miembros menores de 20 y mayores de 30 años de edad, y que se encontraban realizando actividades administrativas en oficina o no hayan sido expuestos a los trabajos operativos.

\section{Instrumentos de recolección de información}

Escala de bienestar psicológico para adultos (BIEPS-A)

Se utilizó la Escala de bienestar psicológico para adultos (BIEPS-A) construida originalmente por Casullo (2002), y validada en el Perú por Domínguez (2014), fue adaptada en una muestra de 222 universitarios en Lima Metropolitana. El instrumento está conformado por 13 ítems los cuales están distribuidos en una escala de puntuación tipo Likert que van desde 3 a 1 ("De acuerdo", "Ni de acuerdo ni en desacuerdo", "En desacuerdo"); dirigida a personas entre los 16 y 64 años. Los resultados que se obtienen de la prueba oscilan entre los 
13 y 39 puntos, los cuales son obtenidos mediante la suma directa de cada uno de los ítems.

La escala cuenta con una validez por análisis factorial confirmatoria hipotetizando el modelo tetrafactorial propuesto inicialmente por Casullo (2002), los resultados arrojaron que los valores examinados fueron adecuados para cada indicador de ajustes absoluto, asimismo, los valores obtenidos en el modelo hipotetizado evidencian un ajuste aceptable al modelo de los cuatro factores original de Casullo, concluyéndose que los factores adaptados se configuran de acuerdo a la escala original. Por otro lado, presenta una confiabilidad de alfa de Cronbach de .966 con respecto a la escala en general, asimismo, con respecto a los cuatro factores con los que cuenta se realizó un análisis de alfa de Cronbach obteniendo en: (1) Aceptación/control de situaciones un valor de .884; (2) autonomía un valor igual a .836; (3) vínculos sociales igual a .871 y (4) proyectos un valor de .908. Los datos de confiabilidad para la presente investigación se encuentran destallados en la tabla 2 de Resultados.

\section{Escala de Calidad de Vida Profesional CVP-35}

Para la recolección de datos de la segunda variable se hizo mediante la Escala de Calidad de Vida Profesional CVP-35 cuyo autor original es Cabezas (1998), adaptada en Perú por Grimaldo y Reyes (2013) en una muestra de 409 alumnos de una escuela de posgrado de Lima. El instrumento está conformado por 35 ítems, contando con una escala de 1 al 10, las cuales corresponden a cuatro categorías entre ellas: (1) nada (1 y 2), (2) algo (3, 4 y 5), (3) bastante (6, 7 y 8$)$ y (4) mucho (9 y 10); puede ser aplicada de manera individual o colectiva entre personas de 16 a 60 años. Con respecto a su corrección se deben de sumar las puntuaciones directas de cada una de las preguntas. Fue expuesto a la validez de contenido mediante el criterio de jueces obteniendo un $\mathrm{p}<.05$ en todos los ítems; con respecto a la validez de constructo, se utilizó el análisis factorial exploratorio mediante el método de extracción de ejes principales y de rotación oblicua obteniendo tres factores extraídos que contenían el $61 \%$ de varianza de los ítems, siendo que estos factores alcanzaban a explicar 
con algún grado de significado conceptual las correlaciones entre los ítems. Por otro lado, con respecto a la confiabilidad fue calculada mediante el coeficiente de alfa de Cronbach obteniendo un valor de .92 en toda la escala. Asimismo, en relación a la confiabilidad de las dimensiones se obtiene que: (1) carga laboral presenta un alfa de .90; (2) motivación intrínseca cuenta con un valor de .91 y (3) apoyo directivo presente un valor de .95 . Además, con respecto a la confiabilidad en el presente estudio se detallan en la tabla 2 de Resultados.

\section{Procedimiento}

Para realizar la presente investigación se solicitó previa autorización por parte de los representantes de distintas comisarías de los distritos que conforman Lima Metropolitana, entre ellas: el distrito de La Molina, Surquillo, Santa Anita, entre otras. Luego se acordó con cada representante, la fecha y hora de aplicación de las pruebas dentro de sus ambientes. En cuanto a la aplicación de los instrumentos se llevó a cabo en su mayoría en horario de formación, las cuales tenían lugar en horarios de la mañana y tarde; asimismo, se llevó a cabo la descripción de los objetivos de la investigación, la participación voluntaria y la confidencialidad de los datos recopilados, para ello se hizo entrega del consentimiento informado (apéndice 1) con la finalidad de constar la participación voluntaria de cada uno de los participantes. Con respecto a la disposición de los participantes, la mayoría de ellos mostraba una actitud colaborativa, porque muchos de ellos mencionaban que su salud mental no era tomada en cuenta en muchas ocasiones.

Previamente a la recolección de datos se llevó a cabo algunas aplicaciones pilotos (10), con la finalidad de que los participantes no presentarán problemas con respecto a la comprensión de los ítems; posteriormente luego que se comprobó la correcta comprensión se procedió a la aplicación masiva de la muestra, siendo en un comienzo un total de 240 miembros de la PNP, sin embargo, 107 fueron descartados ya que no contaban con los criterios de inclusión previamente detallados, quedando 133 como total de la muestra de 
estudio.

\section{Análisis de datos}

Luego de la recolección de los datos mediante los instrumentos previamente detallados, los datos fueron procesados mediante el programa estadístico de Jamovi 1.2.27 Primeramente se procedió a la revisión de la confiabilidad de los instrumentos mediante el Alfa de Cronbach $(\propto)$; en cuanto a la interpretación se aceptaron valores mayores a .65 (Ponterotto \& Ruckdeschel, 2007). Luego se indago sobre la prueba de normalidad (asimetría y curtosis); después se realizó el análisis de los estadísticos descriptivos de cada una de las variables (bienestar psicológico y calidad de vida profesional) y la correlación entre ellas mediante el estadístico de Pearson. Finalmente, se hizo uso del estadístico de la t de Student, con la finalidad de analizar la causalidad entre las variables y los miembros que han sido y no diagnosticados con COVID-19. 


\section{Resultados}

De acuerdo, a la prueba de normalidad, se evidencia puntuaciones $<|3|$ en las variables estudiadas respecto a la curtosis (g2); asimismo, en cuanto a la asimetría (g1) se observa valores $<.25$, correspondiente a un nivel insignificante de asimetría (Tabla 2). Por ello, se considera aceptable usar el estadístico de Pearson, para el análisis correlacional entre las variables en cuestión.

Sin embargo, en cuanto a la confiabilidad de los instrumentos usados en el presente estudio, se observa que dos de las dimensiones del bienestar psicológico (vínculos y proyectos) presentan un nivel bajo de consistencia $(\alpha<.65)$, sin embargo, dos de sus otras dimensiones (autonomía y aceptación/control) presentan un nivel considerado como aceptable de la consistencia ( $\alpha>$.65). A su vez, se procedió a eliminar 3 ítems, siendo el ítem 4 y 9 de la dimensión de autonomía, y el ítem 6 correspondiente a aceptación/control, debido a que afectaban los niveles de confiabilidad del coeficiente de alfa de Cronbach. Por otra parte, las dimensiones correspondientes a la calidad de vida profesional presentan un nivel excelente de consistencia interna: el apoyo directo $(\alpha>.90)$, carga de trabajo y la motivación intrínseca $(\alpha>$.85) (Tabla 2).

Tabla 2

Análisis descriptivo de las dimensiones de Bienestar psicológica y Calidad de vida profesional

\begin{tabular}{rccccc}
\hline & $\alpha$ & $\mathrm{M}$ & $\mathrm{DE}$ & $\mathrm{Gl}$ & $\mathrm{g} 2$ \\
\hline Bienestar psicológico & & & & & \\
Autonomía & 0.644 & 8.54 & 1.10 & -0.69 & 10.3 \\
Vínculos & 0.268 & 8.46 & 0.875 & -0.45 & 1.94 \\
Proyectos & 0.141 & 5.83 & 0.525 & $-1,60$ & 11.1 \\
Aceptación/Control & 0.678 & 5.90 & 0.458 & -3.35 & 43.9 \\
Apoyo directo & 0.917 & 90.7 & 24.3 & 0.00 & -0.330 \\
Cargas de trabajo & 0.891 & 49.3 & 20.5 & 0.00 & -0.921 \\
Motivación intrínseca & 0.846 & 78.3 & 13.9 & -0.02 & 3.73 \\
Calidad de vida profesional & - & 7.56 & 1.67 & -0.03 & -0.754 \\
\hline
\end{tabular}

En la tabla 3 se presenta las puntuaciones obtenidas con respecto a la correlación entre 
las variables de estudio. Debido a los resultados detallados en la anterior tabla, con respecto a la confiabilidad de dos de las dimensiones de la Escala de bienestar psicológico no se procedió con el análisis de correlación de dichas dimensiones.

Al analizar cada dimensión por separado, se encontró que, en el bienestar psicológico, la aceptación/control no guarda relación con la carga de trabajo $(\mathrm{r}=.06)$, la motivación intrínseca $(\mathrm{r}=-.00)$, el apoyo directo $(\mathrm{r}=.02)$ y la calidad de vida $(\mathrm{r}=.80)$, todas las variables presentan un nivel bajo de magnitud del efecto $(r<.20)$, con respecto a las cuatro variables correspondientes a la calidad de vida profesional. Al mismo tiempo, en la autonomía del bienestar psicológico se evidencia que no existe correlación con las variables cargas de trabajo ( $\mathrm{r}=-.12)$, y motivación intrínseca $(\mathrm{r}=.10)$, sin embargo, se puede observar relación con el apoyo directo $(\mathrm{r}=.20)$ y con la calidad de vida $(\mathrm{r}=.45)$, con respecto al primero se puede observar un nivel insignificante y en el segundo un nivel bajo de magnitud del efecto.

Tabla 3

Análisis correlacional entre las dimensiones de Bienestar psicológico y Calidad de vida profesional

\begin{tabular}{llccccc}
\hline & CVP & $\begin{array}{c}\text { Cargas de } \\
\text { trabajo }\end{array}$ & $\begin{array}{c}\text { Motivación } \\
\text { intrinseca }\end{array}$ & $\begin{array}{c}\text { Apoyo } \\
\text { directo }\end{array}$ & $\begin{array}{c}\text { Calidad } \\
\text { de Vida }\end{array}$ \\
\hline BP & & - & - & - & - & - \\
Aceptación/ & Pearson's r & - & - & - & - & - \\
Control & p-valor & - & 0.06 & -0.00 & 0.02 & -0.13 \\
& & & 0.50 & 0.88 & 0.80 & 0.80 \\
Autonomía & $\begin{array}{l}\text { Pearson's r } \\
\text { p-valor }\end{array}$ & - & -0.12 & 0.10 & 0.20 & 0.45 \\
& & - & 0.16 & 0.25 & $0.02 *$ & $0.00^{*}$ \\
\hline
\end{tabular}

$* p<.05$

Por otro lado, se observa en la tabla 4 en cuanto al bienestar psicológico y sus dimensiones que la autonomía $(t[131]=-1.504 ; \mathrm{d}=-.263 ; \mathrm{p}>.05)$ y la aceptación/control $(t[131]=-.126 ; \mathrm{d}=-.022 ; \mathrm{p}>.05)$ no presentan diferencias significativas con respecto a los miembros con y sin diagnóstico de COVID-19, asimismo, se observa un nivel insignificante del efecto $(\mathrm{d}<.41)$. 
En cuanto, a la calidad de vida profesional y sus dimensiones, se observa que en la motivación intrínseca $(t[131]=0.548 ; \mathrm{d}=.096 ; \mathrm{p}>.05)$, el apoyo directo $(t[131]=.111 ; \mathrm{d}=.912$; $\mathrm{p}>.05)$ y la calidad de vida $(t[131]=-1.287 ; \mathrm{d}=-.225 ; \mathrm{p}>.05)$ no presentan diferencias significativas. A excepción de la carga de trabajo, que presenta diferencias $(t[131]=-2.007$; $\mathrm{d}=.351 ; \mathrm{p}<.05)$, observándose que el puntaje es mayor en participantes diagnosticados $(\mathrm{M}=53.28 ; \mathrm{DE}=21.18)$. Todas las pruebas de la $\mathrm{d}$ de Cohen realizadas muestran un tamaño del efecto insignificante $(\mathrm{d}<.41)$, lo que significa que existe mucho error para rechazar la afirmación de los rasgos idénticos (hipótesis nula).

Tabla 4

Comparación del Bienestar Psicológico y la Calidad de Vida Profesional conforme al diagnóstico COVID-19.

\begin{tabular}{|c|c|c|c|c|c|c|c|}
\hline & Dimensiones & $D g^{*}$ & $N$ & Media & $D S$ & $d$ & $p$ \\
\hline \multirow[t]{4}{*}{$\mathrm{BP}$} & \multirow[t]{2}{*}{ Autonomía } & $\mathrm{Si}$ & 58 & 8.38 & 1.35 & \multirow[b]{2}{*}{-0.263} & \multirow[b]{2}{*}{0.135} \\
\hline & & No & 75 & 8.67 & 0.84 & & \\
\hline & \multirow[t]{2}{*}{ Aceptación/control } & $\mathrm{Si}$ & 58 & 5.90 & 0.41 & \multirow{2}{*}{-0.022} & \multirow[b]{2}{*}{0.900} \\
\hline & & No & 75 & 5.91 & 0.50 & & \\
\hline \multirow[t]{8}{*}{$\mathrm{CVP}$} & \multirow[t]{2}{*}{ Cargas de trabajo } & $\mathrm{Si}$ & 58 & 53.28 & 21.18 & \multirow[b]{2}{*}{0.351} & \multirow[b]{2}{*}{$0.047 *$} \\
\hline & & No & 75 & 46.16 & 19.56 & & \\
\hline & \multirow{2}{*}{$\begin{array}{l}\text { Motivación } \\
\text { intrinseca }\end{array}$} & $\mathrm{Si}$ & 58 & 79.05 & 13.06 & \multirow[b]{2}{*}{0.096} & \multirow[b]{2}{*}{0.585} \\
\hline & & No & 75 & 77.72 & 14.51 & & \\
\hline & \multirow[t]{2}{*}{ Apoyo directo } & $\mathrm{Si}$ & 58 & 90.97 & 24.34 & \multirow[b]{2}{*}{0.019} & \multirow[b]{2}{*}{0.912} \\
\hline & & No & 75 & 90.49 & 24.40 & & \\
\hline & \multirow[t]{2}{*}{ Calidad de Vida } & $\mathrm{Si}$ & 58 & 7.34 & 1.56 & \multirow[b]{2}{*}{-0.225} & \multirow[b]{2}{*}{0.200} \\
\hline & & No & 75 & 7.72 & 1.74 & & \\
\hline
\end{tabular}

* Dg: Diagnóstico

*BP: Bienestar psicológico

$* C V$ : Calidad de vida profesional

$*_{p}<0.05$ 


\section{Discusión}

En la actual situación por la que se atraviesa debido a la pandemia del COVID-19, los miembros de la Policía Nacional del Perú (PNP) se han visto expuestos a afrontar una nueva demanda de esfuerzo físico y mental para llevar a cabo el cumplimiento de las medidas de seguridad que se adoptaron en el estado peruano frente a la emergencia sanitaria; como consecuencia muchos de los miembros de la PNP estuvieron expuestos a la perdida de familiares y/o amigos, así como contagios y afecciones psicológicas, como: estrés, depresión y ansiedad. Por lo que en la presente investigación se buscó determinar la relación entre el bienestar psicológico (BP) y calidad de vida profesional (CVP) durante la pandemia de COVID-19 en miembros de la PNP de Lima Metropolitana; dentro de este marco, también se ha optado por realizar un estudio comparativo de estas dos variables en función a la presencia y grado, según del diagnóstico por COVID-19 (con y sin contagio).

Conforme a los resultados obtenidos, cabe mencionar que dos dimensiones de la Escala de bienestar psicológico (vínculos y proyectos) no presentaron un nivel de consistencia adecuada para ser consideradas entre los análisis.

En primer lugar, con respecto a la hipótesis general del presente estudio, las variables de bienestar psicológico y calidad de vida profesional no guardan relación, observándose un tamaño del efecto insignificante, esto podría ser debido a que los miembros de la PNP han desarrollado la resiliencia entre un nivel medio a alto, caracterizados por tener adecuados niveles de ecuanimidad (capacidad de mantener una adecuada postura frente a las adversidades) y perseverancia (mantenerse constante sin importar lo difícil de la situación) (Chavéz, 2019; Ríos, 2019), dichas características permiten que las personas sobrelleven de manera adecuada las situaciones adversas, es decir, que no es necesario presentar un adecuado ambiente físico en el trabajo o variables positivas, como: buen salario, adecuado 
horario de trabajo, reconocimiento por parte de los superiores o adecuadas relaciones interpersonales que influyen en el bienestar psicológico; debido a la naturalidad de las actividades que están expuestos los miembros de la PNP han desarrollado mayor capacidad de adaptación frente a situaciones problemáticas. Sin embargo existen estudios donde contrariamente a los resultados obtenidos, sí se evidencia dicha asociación y el bienestar psicológico está relacionado con una percepción satisfactoria del trabajo, motivación intrínseca, el sentirse apoyado y una buena relación interpersonal (Ballesteros, Medina \& Caycedo 2006; Brancato \& Juri, 2011). Dicha relación se evidencia en el estudio realizado en una muestra de 237 docentes de instituciones públicas en el Callao, donde se obtuvo una relación estadísticamente significativa entre las variables (Rivas, 2019), asimismo, existen evidencias que las malas condiciones de trabajo en sus diferentes áreas, como: ambiental, salarial, horario de trabajo, apoyo del equipo de trabajo y la sobre carga laboral afectan de manera negativa en el bienestar psicológico (Parras \& Otálvaro, 2018; Sander \& Córdoba, 2010). Siendo así, no se puede negar las evidencias empíricas de que los miembros de la PNP están constantemente expuestos a diferentes actividades que ponen en riesgo su bienestar psicológico y calidad de vida profesional, los cuales probablemente se hallan visto afectados por las diferentes crisis por la que atraviesa el Perú, como la crisis sanitaria debido a la pandemia del COVID-19 (Delgado, 2020), la crisis política (Paredes \& Encinas, 2020) y social (Manrique, 2020), ya que son ellos los encargados de garantizar, mantener y reestablecer el orden interno.

En cuanto, a la primera hipótesis especifica planteada se propuso determinar si existe relación significativamente negativa entre el factor de cargas de trabajo de la calidad de vida profesional y el bienestar psicológico (autonomía y aceptación/control), obteniendo como resultado que no existe una relación entre las variables, y un nivel insignificante de la magnitud del efecto; dichos resultados debieron de obtenerse debido a que la autonomía y 
aceptación/control son variables que están relacionadas con aspectos internos de la personalidad como autocontrol, autococimiento y autoaceptación, a diferencia de aspectos externos como el ámbito laboral (Liberal, Escudero, Cantallops \& Ponseti, 2014). Sin embargo, existen evidencias que a mayor cargas de trabajo mayor son las consecuencias negativas para la salud mental de las personas, teniendo consecuencias como depresión y estrés asociada con la baja capacidad de aceptar y afrontar las situaciones complicadas (aceptación/control) (Gonzales, 2016; Meneses, Hernández \& Muñoz, 2019). Asimismo, la carga de trabajo está asociada con el desgaste mental, el desequilibrio emocional, falta de motivación y una baja autoestima, vinculada a la autonomía (Jaramillo, 2014; Marques et al., 2017). La carga de trabajo no ha sido ajena a los miembros de la PNP quienes debido a la pandemia del COVID-19, han experimentado mayor demanda física y mental para poder cumplir sus funciones laborales, y de esa manera resguardar la seguridad de la ciudadanía en general.

Respecto, a la segunda hipótesis especifica se planteó determinar la existencia de relación entre el factor de motivación intrínseca de la calidad de vida profesional y las dimensiones de bienestar psicológico, obteniendo como resultado que no existe relación entre las variables, además se evidencia una magnitud del efecto insignificante. Contrariamente a ello hay estudios que mencionan que la autonomía, el sentirse autosuficiente para ser independientes y la motivación intrínseca están relacionadas, influenciadas por la percepción que cada persona tenga sobre sí misma y sobre las situaciones (Naranjo, 2009). Un estudio realizado por Quiroz (2018) a 81 miembros de la PNP obtuvo que el 62\% indicaron tener un alto nivel de motivación, asimismo, Tejada (2017) obtuvo que un 36\% de 100 miembros de la PNP indicaron un nivel adecuado de motivación, evidenciando que los miembros de la PNP cuentan en su mayoría con un nivel adecuado de motivación intrínseca que los impulsa a desarrollar sus funciones, lo cual se observa reflejado en el día a día, saliendo a las calles a 
cumplir sus obligaciones.

Por otro lado, como tercera hipótesis específica se planteó la existencia de una correlación entre el factor de apoyo directo de la calidad de vida profesional y las dimensiones de bienestar psicológico, obteniendo como resultado una asociación estadísticamente significativa con respecto al apoyo directo y la autonomía, evidenciando un tamaño del efecto bajo; dichos resultados evidencias que un adecuado apoyo por parte de los jefes o supervisores directos de los miembros de la PNP está relacionado con la autonomía, haciendo referencia a la autodeterminación e independencia; dichos resultados son reforzados por estudios donde se ha encontrado que el sentirse apoyado por los jefe directos aumenta la motivación, el buen desempeño y la estabilidad emocional (Zuazo, 2018; Rodríguez, Blanco, Pérez, García \& Gayoso, 2005). Asimismo, la calidad de vida profesional está relacionada con la autonomía de bienestar psicológico, con un nivel bajo en la magnitud del efecto, los resultados indican que las buenas prácticas en las distintas áreas del trabajo permiten el mejor desarrollo de habilidades, actitudes y promueve la autonomía de los miembros de la PNP (Granados, 2011).

Adicionalmente, se plantearon dos hipótesis comparativas, la primera fue la existencia de diferencias entre el bienestar psicológico entre los miembros del PNP con y sin diagnóstico de COVID-19 y el segundo fue la existencia de diferencias entre la calidad de vida profesional entre los miembros del PNP con y sin diagnóstico de COVID-19, encontrando como resultado en la mayoría de los casos que no existe diferencias entre los grupos de estudio, es decir, que se evidencia que el contar o no con el diagnóstico positivo de COVID-19 no influye de manera significativa en el bienestar psicológico y calidad de vida profesional de los miembros de la PNP, dichos datos obtenidos pueden deberse a que los miembros de la PNP cuentan con un nivel de resiliencia de medio a alto como se detalló posteriormente, además, dichos profesionales se caracterizan por presentar niveles de medios 
a altos con respecto a la motivación laboral, haciendo referencia al impulso por ejercer las actividades laborales guiados por diferentes factores internos y externos (Tejada, 2017); y altos niveles de responsabilidad, que involucran ejercer sus funciones aún a pesar de las distintas adversidades que se presenten en la práctica de la misma (Holgado, 2018). Por otro lado, a excepción de cargas de trabajo correspondiente a la calidad de vida profesional, se evidencia una diferencia estadísticamente significativa entre los miembros de la PNP que han sido diagnosticados con COVID-19, con un tamaño del efecto insignificante, es decir, que los miembros de la PNP que ha sido diagnosticados positivamente con COVID-19 perciben una mayor carga de trabajo con un nivel insignificante de diferencia; sin embargo dichos resultados se refuerzan con los hallazgos en algunos estudios realizados en pacientes que han superado el COVID-19, dichos estudios afirman que posterior a la recuperación la persona presentan secuelas psicológicas, como: alteraciones en la percepción de las distintas experiencias, ansiedad, agotamiento físico y/o mental y desequilibrio emocional (Llorens, 2020; Tomé, 2020), motivo por el cual los miembros con diagnóstico positivo de COVID-19 hayan percibido mayor demanda de trabajo a diferencias de los que no cuentan con el diagnóstico positivo de COVID-19.

Con respecto a las limitaciones que se presentaron durante el desarrollo de la presente investigación, se encontraron algunas, entre ellas: sesgo de la selección de la muestra al no estar basado en una población general y al usar un muestreo no aleatorio y por convivencia, debido a la disponibilidad de la muestra ya que los miembros de la PNP empiezan sus labores a primaras horas de la mañana es difícil encontrar a un grupo mayoritario durante las visitas a las comisarias; asimismo, cabe mencionar que algunas aplicaciones de los instrumentos se llevaron a cabo en la vía pública, donde existían distintos tipos de estímulos distractores como: ruido de los vehículos de transporte, movimiento, etc., ello pudo afectar en la atención prestada al desarrollo de los ítems; además, en algunas ocasiones se evidenciaba poco interés 
en participar de la investigación por parte de algunos líderes (alférez) encargados de la formación de los miembros de la PNP, quienes presionaban a sus compañeros a que desarrollen rápidamente las pruebas; por otro lado, también se evidencia la falta de estudios correlacionando ambas variables en cuestión, por lo que dificulta comprar lo resultados encontrados. Asimismo, no se descarta la presencia de deseabilidad social por parte de los participantes, que puedo influir en las respuestas de las ítems de prueba; también, existe la posibilidad que no hayan respondido con honestidad con respecto a su diagnóstico de COVID-19, el cual podría deberse a la deseabilidad social ante el miedo al rechazo por las personas de su círculo social, debido a que ante las amenaza de una enfermedad las personas que pertenecen a un mismo círculo social suelen ser más estrictas al momento de la inclusión, asimismo, pasa cuando un extraño quiere incluirse al mismo círculo social, a dicha actitud asumida por el grupo social se le denomina estigma social (Domínguez, Aguilera, Acosta, Navarro \& Ruiz, 2012; Cedeño, 2019). Finalmente, otra de las limitaciones encontrada es el nivel débil de consistencia interna en dos de las dimensiones de la Escala de bienestar psicológico, lo que estaría influyendo de manera negativa con respecto a la objetividad del presente estudio.

\section{Conclusiones:}

Teniendo en cuenta los resultados obtenidos se llega a la conclusión que no existe una correlación entre las variables en cuestión para la presente muestra de estudio, asimismo, se evidencia que la variable aceptación/control correspondientes al bienestar psicológico no se relaciona de manera significativa con las dimensiones de calidad de vida profesional (Carga de trabajo, motivación intrínseca, apoyo directo y calidad de vida); asimismo, con respecto a la variable autonomía, se observa la falta de correlación con cargas trabajo, motivación intrínseca y apoyo directo, aunque se discrepan de los hallazgos en estudios previos, los 
cuales evidencian que el bienestar psicológico está relacionado a con variables a nivel personal y social, entre ellas el autocontrol, autoconocimiento y autoaceptación (Hernández \& García, 2020; Liberal, et al., 2014), y las adecuadas condiciones ambientales, como la relaciones familiares positivas y el sentirse apoyado por sus relaciones interpersonales y un adecuado ámbito laboral (Ballesteros, Medina \& Caycedo, 2006). Por otro lado, autonomía, presenta una correlación estadísticamente significativa con calidad de vida, con un tamaño del efecto bajo, los cuales son corroborados con estudios previos.

Por otro lado, se concluye que los miembros de la PNP con y sin diagnóstico no están asociados al bienestar psicológico y calidad de vida profesional, evidenciando que las características, halladas en previos estudios, de los miembros de la PNP se evidencian niveles de medios a altos de resiliencia que los ayudan afrontar de manera adecuada la presente situación adversa por la que se atraviesa a nivel nacional (Chavéz, 2019; Ríos, 2019). Por otro lado, se concluye que si existe diferencia estadísticamente significativa entre los que han sido o no diagnosticados con COVID-19 respecto a la carga de trabajo, evidenciándose que los que han sido diagnosticados perciben una mayor carga de trabajo, debido a que posterior a la recuperación el $56 \%$ de los pacientes con COVID-19 presentan algunas secuelas psicológicas, como: alteraciones en la percepción de las distintas experiencias, ansiedad, agotamiento físico y/o mental y desequilibrio emocional (Gennaro, et al., 2020; Llorens, 2020; Tomé, 2020).

\section{Recomendaciones}

Tomando en cuenta los resultados obtenidos en el presente estudio, se propone que se lleve a cabo una investigación analizando la correlación de ambas variables en una muestra representativa de la población, asimismo, debe de tenerse en cuenta un adecuado ambiente de aplicación de las pruebas para evitar que los estímulos distractores interfieran en el desarrollo 
de las pruebas, también, debe de cuestionarse la confiabilidad y validez de la Escala de Bienestar Psicológico en una muestra de miembros de la PNP o de los contrario es recomendable hacer uso de otra escala para una mayor objetividad de los resultados, además, debe de tenerse en cuenta que el presente estudio fue realizado en distintos distritos correspondiente a Lima centro, norte, este y sur; sin embargo, según el INEI (Instituto Nacional de Estadística e Informática, 2018) menciona que los distritos con mayor índice de delincuencia son los Distritos de Los Olivos, San Juan de Lurigancho y Cercado de Lima, ello podría indicar que los miembros de la PNP que laboren en dichas zonas reciben una mayor presión con respecto a mantener constantemente su estado de alerta frente al peligro a diferencia de lo que laboran en otros distritos con bajo índice de delincuencia; también debería de tomarse en cuenta la infraestructura (disponibilidad de ambientes adecuados para el desarrollo de las funciones asignadas, servicios básicos, etc.) de las 126 comisarías de Lima (54.6\% de las comisarias cuentan con un techo de concreto), número de población que se atiende por comisaria (el 38.9\% de las comisarías de Lima atiende de 80001 a más habitantes), el equipamiento (solo el $45.2 \%$ de las comisarías en Lima cuentan con al menos una computadora propia y con menos de 3 años de uso) y el factor humano (INEI, 2016; INEI, 2017) estos datos podrían ser muy importantes de considerar al momento de la recolección de datos para separar las muestras según corresponda. Además, debe de tenerse en cuenta que los constantes cambios por los que atraviesa la sociedad están desarrollando un entorno complejo, donde las estrategias de abordaje del bienestar psicológico y calidad de vida laboral están enlazadas y deben de ser considerados para evitar problemas de salud mental y laboral (Durán, 2010). Asimismo, se propone realizar talleres de intervención con respecto a la calidad de vida profesional (involucrando aspectos físicos propias de la labor así como personales) con la finalidad de mejorar los diferentes aspectos que involucran lograr el equilibrio de una adecuada calidad de vida dentro del ámbito laboral como mejorar las 
habilidades relacionadas con las relaciones interpersonales y la motivación intrínseca; y de esa manera conseguir adecuados resultados con respecto al bienestar psicológico; además es importante el desarrollo de talleres que permitan no solo brindar conocimiento sobre las consecuencias físicas que produce el COVID-19, si no también brindar talleres relacionados al reconocimiento y manejo de las emociones que se relacionan con la pandemia, asimismo, brindar talleres grupales brindando espacios donde los miembros de la PNP puedan expresar sus experiencia vividas durante la pandemia permitiendo de esa manera reducir el impacto negativo que causa la pandemia por COVID-19 en la salud mental de estos profesionales, debido a que representan a una de las instituciones que lucha en primera línea frente al COVID-19. 


\section{Referencias}

Ato, M., López, J. \& Benavente, A. (2013). Un sistema de clasificación de los diseños de investigación en psicología. Anales de psicología,29(3), 1038-1059. doi: http://dx.doi.org/10.6018/analesps.29.3.178511

Ballesteros, B., Medina, A. \& Caycedo, C. (2006). El bienestar Psicológico definido por asistentes a un servicio de consulta psicológica en Bogotá, Colombia. Univ. Psychol. Bogota, 5(2), 239-258. Recuperado de http://www.scielo.org.co/pdf/rups/v5n2/v5n2a04.pdf

Balluerka, N., Gómez, J., Hidalgo, M., Gorostiaga, A., Espada, J. Padilla, J. \& Santed, M. (2020). Las consecuencias psicológicas de la Covid-19 y el confinamiento. Servicios de publicaciones de la Universidad del país de Vasco. Obtenido de https://www.ub.edu/web/ub/ca/menu_eines/noticies/docs/Consecuencias_psicologicas_CO VID-19.pdf

Brancato, B. \& Juri, F. (2011). ¿Puede influir el clima laboral en la productividad. Universidad Nacional de Cuyo. Mendoza. Recuperado de https://bdigital.uncu.edu.ar/objetos_digitales/4936/brancatopuedeinfluirclimalaboralenlaproductividad.pdf

Cabezas, C. (1998). Síndrome de desgaste profesional, estrés laboral y calidad de vida profesional. FMC: Formación Médica Continuada en Atención Primaria, 5(8), 491-492.

Casullo, M. (2002). Evaluación del bienestar psicológico en Iberoamérica. Buenos Aires: Paidós.

Caycho, T., Carbajal, C., Vilca, L., Heredia, J. \& Gallegos, M. (2020). COVID-19 y salud mental en policías peruanos: resultados preliminares. Acta Med Perú, 37(3), 396-8. doi: https://doi.org/10.35663/ amp.2020.373.1503

Cedeño, A. (2019). La estigmatización: una forma normalizada de la violencia intragénero. Universidad $y \quad$ Sociedad, 11(4), 77-85. Recuperado de http://scielo.sld.cu/pdf/rus/v11n4/2218-3620-rus-11-04-77.pdf

Chávez, C. (2015). Calidad de vida y bienestar psicológico en personas con discapacidad motriz de un centro de integración de discapacitados de Trujillo (Tesis de Licenciatura). Universidad Privada Antenor Orrego. Recuperado de http://scielo.sld.cu/pdf/rus/v11n4/2218-3620-rus-11-04-77.pdf

Chavez, C. (2019). Resiliencia en efectivos policiales pertenecientes a la División de Inteligencia de la Región Policial de Lima. (Tesis de licenciatura en Psicología). 
Universidad Inca Garcilaso de la Vega. Obtenido de http://repositorio.uigv.edu.pe/bitstream/handle/20.500.11818/4100/008594_Trab_Suf_Prof Chavez\%20Carranza\%20Camilo.pdf? sequence $=2 \&$ is Allowed $=y$

Colegio de Sociólogos del Perú (2020). El coronavirus y su impacto en la sociedad actual y futura. Recuperado de https://colegiodesociologosperu.org.pe/wp-content/uploads/ElCoronavirus-y-su-impacto-en-la-sociedad-actual-y-futura-mayo-2020.pdf

Decreto de Urgencia N 012, Diario Oficial El Peruano, Lima, Perú, 16 de enero de 2020. Recuperado de https://busquedas.elperuano.pe/download/url/decreto-de-urgencia-queestablece-medidas-temporales-para-el-decreto-de-urgencia-n-012-2020-1846271-2

Decreto Supremo N 044-PCM, Diario Oficial El Peruano, Lima, Perú, 15 de marzo de 2020. Recuperado de https://cdn.www.gob.pe/uploads/document/file/566448/DS044PCM_1864948-2.pdf

Decreto Supremo N 094-PCM, Diario Oficial El Peruano, Lima, Perú, 23 de mayo de 2020. Recuperado de https://cdn.www.gob.pe/uploads/document/file/730522/DS_N_094-2020PCM.pdf

Decreto Supremo N ${ }^{\circ}$ 156-PCM, Diario Oficial El Peruano, Lima, Perú, 26 de setiembre de 2020. Recuperado de https://busquedas.elperuano.pe/download/url/decreto-supremo-quemodifica-el-decreto-supremo-n-116-2020-decreto-supremo-n-156-2020-pcm-1888160-2

Delgado, D. (2020). La COVID-19 en el Perú: una pequeña tecnocracia enfrentándose a las consecuencias de la desigualdad. Asociación Carolina, 1-16. doi: https://doi.org/10.33960/AC_26.2020

Domínguez, A, Aguilera, T., Acosta, T., Navarro G. \& Ruiz, Z. (2012). La deseabilidad social revalorada: más que una distorsión, una necesidad de aprobación social. Revista UNAM, 2(3), 808-824. Recuperado de http://www.scielo.org.mx/pdf/aip/v2n3/v2n3a5.pdf

Domínguez, S. (2014). Análisis Psicométrico de la Escala de Bienestar Psicológico para Adultos en estudiantes universitarios de Lima: un enfoque de ecuaciones estructurales. Psychologia. Avances de la disciplina,8(1), 23-31. Recuperado de https://www.redalyc.org/pdf/2972/297231283003.pdf

Durán, M. (2010). Bienestar psicológico: el estrés y la calidad de vida en el contexto laboral. Revista Nacional de Administración, 1(1), 71-84. Recuperado de file:///D:/DESCARGAS/Dialnet-BienestarPsicologico-3698512\%20(1).pdf

El Peruano (15 de agosto de 2020). Unos 400 policías y 125 médicos han muerto en Perú por Covid-19.

Friedman, E., Hayney, M., Gayle, L., Burton, S. \& Ryff, C. (2007). La interleucina-6 plasmática y los receptores solubles de IL-6 están asociados con el bienestar psicológico de las mujeres que envejecen. Psicología de la salud, 26(3), 305313.doi: https://doi.org/10.1037/0278-6133.26.3.305 
García, D., Hernández, J., Espinosa, J. \& Soler, M. (2020). Salud mental en la adolescencia montevideana: una mirada desde el bienestar psicológico. Recuperado de https://bonga.unisimon.edu.co/bitstream/handle/20.500.12442/6341/PDF.pdf?sequence=1 \&isAllowed $=\mathrm{y}$

García, F., Manquián, E. \& Rivas, G. (2016). Bienestar psicológico, estrategias de afrontamiento y apoyo social en cuidadores informales. Psicoperspectivas, 15(3), 101-111. Recuperado de https://scielo.conicyt.cl/pdf/psicop/v15n3/art10.pdf

Granados, I. (2011). Calidad de vida laboral: historia, dimensiones y beneficios. Revista IIPSI, $\quad$ 14(2), 271-276. $\quad$ Recuperado de http://200.62.146.34/bitstream/handle/123456789/4267/revista_de_investigacion_en_psico $\underline{\text { logia13v14n2 2011.PDF? sequence }=1 \& \text { is Allowed }=\mathrm{y}}$

Gennaro, F., Pizzol, D., Marotta, C., Antunes, M., Racalbuto, V., Veronese, N. \& Smith, L. (2020). Coronavirus diseases (COVID-19) current status and future perspectives: a narrative review. International journal of environmental research and public health, 17(8), 2690. doi: https://doi.org/10.3390/ijerph17082690

Grimaldo, M. \& Reyes, M. (2013). Calidad de vida profesional y sueño en profesionales de Lima. Revista latinoamericana de psicología,47(1), 50-57. Recuperado de https://www.sciencedirect.com/science/article/pii/S0120053415300066

Grimaldo, P. (2010). Calidad de vida y estilo de vida saludable en un grupo de estudiantes de posgrado de la ciudad de Lima. Pensamiento psicológico, 8(15).

Gonzales, I., Dos Santos, E., Paiva, L., Aparecida, L., Aparecida, R. \& Alcalá, D. (2016). Ansiedad, depresión, resiliencia, y autoestima en individuos con enfermedades cardiovasculares. Revista Latino-Americana de Enfermagem, 24, 1-10. doi: 10.1590/1518$\underline{8345.1405 .2836}$

Hernández, R., Fernández, C. \& Baptista, P. (2014). Metodología de la investigación (Sexta Edición). México. Editorial Mc Graw Hill.

Hernandez, J., López, C., Portolés, A., Muñoz, A. \& Mendoza, Y. (2017). Bienestar psicológico, personalidad y actividad Física. Un estilo de vida para la vida adulta. Acción Psicológica, 14(1), 65-78. doi: http://dx.doi.org/10.5944/ap.14.1.19262

Holgado, D. (2018). Autoestima y resiliencia de los estudiantes de educación superior de la Escuela Técnica Superior Policía Nacional de Perú Alférez, Mariano Santos Mateo ciudad del Cusco. (Tesis de maestría en Psicología). Universidad Cesar Vallejo. Obtenido de https://repositorio.ucv.edu.pe/bitstream/handle/20.500.12692/37607/holgado_tl.pdf?seque $\underline{\text { nce }=1 \& \text { is Allowed }=y}$ 
Hun, N., Urzúa, A., López, A., Escobar, N. \& Leiva, J. (2019). Comportamiento alimentario y bienestar psicológico en población universitaria en el norte de Chile. Revista de la Sociedad Latinoamericana de Nutrición, 69(4). https://www.researchgate.net/profile/Nelson_Hun/publication/342734083_Comportamient o_alimentario_y_bienestar_psicologico_en_poblacion_universitaria_en_el_norte_de_Chil e/links/5f15ab5a92851c1eff219bc7/Comportamiento-alimentario-y-bienestar-psicologicoen-poblacion-universitaria-en-el-norte-de-Chile.pdf

Instituto Nacional de Estadísticas e Informática (INEI). Análisis de la Información de Infraestructura y Equipamiento de las Comisarias, 2016. Recuperado de https://www.inei.gob.pe/media/MenuRecursivo/publicaciones_digitales/Est/Lib1461/cap0 2.pdf

Instituto Nacional de Estadísticas e Informática (INEI). Anuario estadístico de criminalidad y $\begin{array}{llll}\text { seguridad ciudadana } & \text { 2011-2017. }\end{array}$ https://www.inei.gob.pe/media/MenuRecursivo/publicaciones_digitales/Est/Lib1534/libro. pdf

Instituto Nacional de Estadísticas e Informática (INEI). Indicadores del VI Censo Nacional de Comisarías, 2017. Recuperado de https://www.inei.gob.pe/media/MenuRecursivo/publicaciones_digitales/Est/Lib1528/cap0 4.pdf

Jaramillo, J. (2015). Incidencia de la carga laboral en el rendimiento de los colaboradores del área de producción de la empresa prologic S.A. de la ciudad de Guayaquil. (Tesis de licenciatura). Escuela superior politécnica del litoral de Ecuador. Recuperado de http://www.dspace.espol.edu.ec/xmlui/handle/123456789/35649

Ledesma, R., Macbeth, G. \& Cortada (2008). Tamaño del efecto: revisión teórica y aplicaciones con el sistema estadístico Vista. Revista Latinoamérica, 40(3), 425-439. Recuperado de https://www.redalyc.org/pdf/805/80511493002.pdf

Liberal, R., Escudero, J., Cantallops, J. \& Ponseti, J. (2014). Impacto psicológico de las lesiones deportivas en relación al bienestar psicológico y la ansiedad asociada a deportes de competición. Revista de Psicología del Deporte, 23(2), 451-456. Recuperado de https://www.redalyc.org/pdf/2351/235131674026.pdf

Llorens, D. (11 de agosto de 2020). Más de la miad de las personas que se recuperan de la Covid-19 tienen secuelas psicológicas. Saber tve Vivir.

Marques, S., Martins, F., Almeida, L., Rodrigues, Z., Alves D. \& Souza, F. (2017). Accidente de trabajo y autoestima de profesionales de enfermería en ambientes hospitalarios. Revista 
Latino-Americana de Enfermagem, 25, 1-8. doi: 10.1590/1518-8345.1632.2872

Manrique, J. (2020). Reflexiones acerca del reconocimiento de nuevos derechos fundamentales en la pandemia sanitaria. Revista de la Facultad de Derecho de México, 70(278-2), 797-814. doi: http://dx.doi.org/10.22201/fder.24488933e.2020.2782.77492

Matalinares, M., Díaz, G., Raymundo, O., Baca, D., Uceda, J. \& Yaringaño, J. (2016). Afrontamiento del estrés y bienestar psicológico en estudiantes universitarios de Lima y Huancayo. Persona, (019), 105-126. Recuperado de file:///D:/DESCARGAS/975Texto\%20de1\%20art\%C3\%ADculo-3247-1-10-20170217\%20(1).pdf

Mayordomo, T., Sales, A., Satorres, E. \& Meléndez, J. (2016). Bienestar psicológico en función de la etapa de vida, el sexo y su interacción. Pensamiento psicológico, 14 (2), 101 112. doi: 10.11144/Javerianacali.PPSI14-2.bpfe

Meneses, D., Hernández M. \& Muñoz D. (2019). Factores percibidos como caga laboral por colaboradores de contact center de la empresa Colsubsidio. (Trabajo de investigación para el diplomado en gerencia del talento humano). Universidad Cooperativa de Colombia. Recuperado de https://repository.ucc.edu.co/bitstream/20.500.12494/13705/1/2019_factores_carga_labora 1.pdf

Mesa, M., Pérez, J., Nunes, C. \& Menéndez, S. (2019). Bienestar psicológico en las personas mayores no dependientes y su relación con la autoestima y la autoeficacia. Scielo. doi: $\underline{10.1590 / 1413-81232018241.35302016}$

Ministerio de Salud (2020). Cuidado de la salud mental del personal de la salud en el contexto del Covid-19. Recuperado de http://bvs.minsa.gob.pe/local/MINSA/5000.pdf

Mjosund, N., Norheim, I., Erikson, M. \& Keyes, C. (2015). Mental health as perceived by persons with mental dosirders-interpretative phenomenological analysis study. ResearchGate, 37-41. Recuperado de file://D:/DESCARGAS/Mjosundetal2015.pdf

Moya, P., Caro, J. \& Monsalves, M. (2017). Percepción de la calidad de vida profesional de docentes de odontología. Revista de Educación Científica, 14(2), 96-104. Recuperado de http://www2.udec.cl/ofem/recs/anteriores/vol1422017/artinv14217a.pdf

Muratori, M., Zubieta, E., Ubillos, S., González, J. \& Bobowik, M. (2015). Felicidad y bienestar psicológico: estudio comparativo entre Argentina y España. Psykhe (Santiago), 24(2), 1-18. doi:10.7764/psykhe.24.2.900

Naranjo, M. (2009). Motivación: perspectiva teóricas y algunas consideraciones de su importancia en el ámbito educativo. Revista Educación, 33(2), 153-170. Recuperado de 
https://www.redalyc.org/pdf/440/44012058010.pdf

Otzen, T. \& Manterola, C. (2017). Técnicas de Muestreo sobre una Población a Estudio. International journal of morphology, 35(1), 227-232.

Paredes, M. \& Encinas, D. (2019). Crisis política y salida institucional. Revista de ciencia política, $\quad 1,28.2$ Recuperado de https://www.researchgate.net/publication/343982337_Peru_2019_crisis_politica_y_salida institucional

Parras, S. \& Otálvaro, A. (2018) el bienestar psicológico y la productividad en el ámbito laboral. Revista Psyconex, 16(10), 1-15. Recuperado de file://D:/DESCARGAS/334751Texto\%20de1\%20art_culo-154466-1-10-20180806.pdf

Pineda, C., Castro, J. \& Chaparro, R. (2018). Estudio psicométrico de las Escalas de Bienestar Psicológico de Ryff en adultos jóvenes colombianos. Pensamiento Psicológico, 16(1), 45-55. doi:10.11144/Javerianacali.PPSI16-1.epeb

Ponterotto, J. \& Ruckdeschel, D. (2007). An overview of coefficient alpha and a reliability matrix for estimating adequacy of internal consistency coefficients with psychological research measures. Perceptual and motor skills, 105(3), 997-1014.

Quiroz, S. (2018). Motivación laboral y engagement del personal de la Policía Nacional del Perú. (Tesis de Licenciatura). Universidad Señor de Sipán.

Rangel, Y., Mayorga, D., Peinado, J. \& Barrón Luján, J. (2017). Actividad física, autoconcepto físico y bienestar psicológico en estudiantes universitarias mexicanas. Revista de psicología del deporte, 26(2), 61-69. Recuperado de https://digibug.ugr.es/bitstream/handle/10481/52798/Rangel\%20Ledezma_Physical\%20ac tivity $\% 20$ habits.pdf? sequence $=1 \&$ isAllowed $=y$

Ríos, M. (2019). Rasgos de personalidad en los policías de la comisaría de Moyobamba del departamento de San Martín. (Tesis de Licenciatura en Psicología). Universidad Inca $\begin{array}{lllll}\text { Garcilaso de la } & \text { Vbtenido }\end{array}$ http://repositorio.uigv.edu.pe/bitstream/handle/20.500.11818/4564/TRABSUFICIENCIA $\underline{\text { RIOS_MARIA.pdf? sequence }=1 \& \text { isAllowed }=\mathrm{y}}$

Rivas, S. (2019). Calidad de vida laboral y bienestar psicológico en docentes de instituciones educativas públicas de Carmen de la Legua. (Tesis de licenciatura en Psicología). Universidad Cesar Vallejo

Rodríguez, F., Blanco, M., Pérez, S., García, L. \& Gayoso, D. (2005). Relación de la calidad de vida profesional y el burnout en médicos de atención primaria. Revista ELSEVIER, 36(8), 442-447. doi: https://doi.org/10.1157/13081058 
Ryan, R. \& Deci, E. (2001). On happiness and human potentials: A review of research on hedonic and eudaimonic well-being. Annual review of psychology, 52(1), 141-166. Recuperado de t: https://www.researchgate.net/publication/12181660

Ryff, C. (1989). Happiness is everything, or is it? Explorations on the meaning of psychological well-being. Journal of Personality \& Social Psychology, 57(6), 1069-1081. doi: https://doi.org/10.1037/0022-3514.57.6.1069

Ryff, C. \& Keyes, C. (1995). The structure of psychological well-being revisited. Journal of personality and social psychology, 69(4), 719. Recuperado de https://www.researchgate.net/profile/Corey_Keyes/publication/15726128_The_Structure_ of_Psychological_Well-Being_Revisited/links/0deec52b1fc8bbbd0a000000.pdf

Salgado, J. \& Leria, F. (2017). Síndrome de burnout y calidad de vida profesional percibida según estilos de personalidad en profesores de educación primaria. Revista CES Psicología, 11(1), 69-89. doi: http://dx.doi.org/10.21615/ cesp.11.1.6

Sander, A. \& Córdoba, E. (2010). Bienestar psicológico labora: su relación con el clima sociolaboral y las condiciones de trabajo. X Congreso Internacional de investigación $Y$ Practicas profesional en Psicología XXV jornadas de investigación XIV Encuentro de investigadores en Psicología del MERCOSUR. Facultad de Psicología - Universidad de Buenos Aires, Argentina.

Somoray, K., Shakespeare-Finch, J. \& Armstrong, D. (2017). The impact of personality and workplace belongingness on mental health workers' professional quality of life. Australian Psychologist, 52(1), 52-60. Recuperado de https://eprints.qut.edu.au/87795/1/Somoray.SF.Armstrong.final.pdf

Tejada, M. (2017). Motivación y satisfacción laboral de los suboficiales de la PNP de tránsito (Tesis de maestría). Universidad Cesar Vallejo, Lima.

Tello, J. (2018). Calidad de vida y bienestar psicológico en estudiantes de psicología de una Universidad Nacional de Lima Metropolitana. (Tesis de licenciatura). Universidad Federico Villa Real.

Tomé, R. (29 de julio de 2020). Superar el coronavirus: ¿Cómo encarar las secuelas psicológicas? El Confidencial.

Torres, L. \& Bahamón, M. (2020). Calidad de vida en jóvenes universitarios. Revista Archivos Venezolanos de Farmacología y Terapéutica, 1-6. Recuperado de https://www.researchgate.net/publication/343022625_Calidad_de_vida_en_jovenes_unive $\underline{\text { rsitarios }}$

Torres, S., Olivella, G., Cudris-Torres, L., Bahamón, M. \& Gil, A. (2020). Programas, 
estrategias y protocolos de bienestar psicológico implementados en jóvenes universitarios.

Recuperado

de

https://bonga.unisimon.edu.co/bitstream/handle/20.500.12442/6199/Programas_Estrategia

s_Protocolos.pdf? sequence $=1 \&$ isAllowed $=\mathrm{y}$

Uribe, A., Ramos, I., Villamil, I. \& Palacio, J. (2018). La importancia de las estrategias de afrontamiento en el bienestar psicológico en una muestra escolarizada de adolescentes. Psicogente 21(4), 440-457 doi: https://doi.org/10.17081/psico.21.40.3082

Urry, H., Nitschke, J., Dolski, I, Jackson, D., Kim, D., Corrina, J., Rosenkranz, M., Ryff, C. \& Singer, B. (2004). Making a life worth living. Psychological Science, 15 (6). Recuperado de https://centerhealthyminds.org/assets/filespublications/UrryMakingPsychologicalScience.pdf

Vargas (2020). Impacto de la epidemia del coronavirus (COVID-19) en la salud mental del personal de salud y en la población general de China. Revista Neuropsiquiatría, 83(1), 5156. doi: https://doi.org/10.20453/rnp.v83i1.3687

Vázquez, C. \& Castilla, C. (2007). Emociones positivas y crecimiento postraumático en el cáncer de mama, Psicooncología, 4 (2), 385-404. Recuperado de https://core.ac.uk/download/pdf/38820052.pdf

Vázquez, C., Hervás, G. Rahona, J. \& Gómez, D. (2009). Bienestar psicológico y salud: aportaciones desde la psicológica positiva. Anuario de Psicología Clínica y de la Salud. 5, 15-28. Recuperado de http://institucionales.us.es/apcs/doc/APCS_5_esp_15-28.pdf

Zuazo, A. (2018). Burnout y calidad de vida profesional en policías de Lima Metropolitana. (Tesis para optar el grado de Licenciado en Psicología). Universidad Peruana de Ciencias Aplicadas (UPC), Lima, Perú. 


\section{Apéndice 1 \\ CONSENTIMIENTO INFORMADO}

Estimada (o) participante:

La investigación titulada "Bienestar psicológico y calidad de vida profesional durante la pandemia por covid-19 en miembros de la Policía Nacional del Perú de Lima Metropolitana” es desarrollada por Mariella Jesús Trujillo Naupari, bachiller en Psicología perteneciente a la Universidad San Ignacio de Loyola.

El presente estudio se realizará en miembros de la PNP que laboren en Lima Metropolitana, con el objetivo de evaluar cómo la actual situación sanitaria por la que se atraviesa a nivel nacional ha afectado el bienestar psicológico y la calidad de vida profesional. Por ello, su participación es esencial en el presente proceso y estaré agradecida si accede a participar.

La duración de toda la evaluación no será mayor a 10 minutos. Se solicitará datos personales como: edad, sexo, estado civil, etc. Así como la respuesta a encuestas que evalúan aspectos de bienestar psicológico y calidad de vida profesional. La información recopilada se usará con fines de estudio y sin ningún otro propósito.

El proceso es completamente voluntario y puede interrumpir su participación cuando lo considere pertinente. Asimismo, cabe mencionar que la información será recogida de forma anónima, por lo que se mantendrá la confidencialidad.

Si desea mayor información del proyecto puede enviar un mensaje al siguiente correo: mariella.trujillo@usil.pe

Si usted está de acuerdo en participar, puede firmar el presente documento.

Firma de la investigadora:

Participante:

Firma:.

Firma de la investigadora:

Firma:

Participante:

Firma: 
Apéndice 2

INFORMACIÓN PERSONAL

Edad:

Sexo:

$\square$ Mujer

$\square$ Hombre

$\square$ Prefiero no decir

Fecha de hoy:

Lugar de Nacimiento:

Lugar de Residencia Actual:

Distrito donde labora

actualmente:

Horario de Trabajo:
$\square 24 \times 24$
$\square$ 48x48
$\square 8$ horas
$\square$ Otro

Tipo de actividad que realiza:

$\square$ Administrativa

$\square$ Operativa

\section{Estado civil}

Soltero

$\square$ Casado

$\square$ Conviviente

$\square$ Divorciado / Separado
Cuál es el grado al que

pertenece actualmente:

Indique sus años de

servicio:

¿En qué tipo de Comisaria labora?

$\square \mathrm{A}$

$\square \mathrm{B}$

$\square \mathrm{C}$

$\square \mathrm{D}$

$\square \mathrm{E}$

¿Ha sido diagnosticado con COVID-19?

$\square$ Sí.

$\square$ No.

De ser afirmativa su respuesta anterior, ¿Cuánto tiempo estuvo en cuarentena?

En general, ¿actualmente se encuentra satisfecho con su trabajo?

$\square$ Sí.

$\square$ No. 


\section{Apéndice 3}

\section{$\underline{\text { ESCALA BIEPS-A (Adultos) }}$}

Le pedimos que lea con atención las frases siguientes. Marque su respuesta en cada una de ellas sobre la base de lo que pensó y sintió durante el último mes. Las alternativas de respuesta son: ESTOY DE ACUERDO - NI DE ACUERDO NI EN DESACUERDO ESTOY EN DESACUERDO. No hay respuestas buenas o malas, todas sirven. No deje frases sin responder. Marque su respuesta con una cruz (aspa) en uno de los tres espacios.

\begin{tabular}{|c|c|c|c|}
\hline & De acuerdo. & $\begin{array}{l}\text { Ni de acuerdo, ni } \\
\text { en desacuerdo. }\end{array}$ & $\begin{array}{l}\text { En } \\
\text { desacuerdo. }\end{array}$ \\
\hline \multicolumn{4}{|l|}{$\begin{array}{l}\text { 1. Creo que sé lo que quiero hacer con mi } \\
\text { vida. }\end{array}$} \\
\hline \multicolumn{4}{|l|}{$\begin{array}{l}\text { 2. Si algo me sale mal puedo aceptarlo, } \\
\text { admitirlo. }\end{array}$} \\
\hline \multicolumn{4}{|l|}{ 3. Me importa pensar que haré en el futuro. } \\
\hline \multicolumn{4}{|l|}{$\begin{array}{l}\text { 4. Puedo decir lo que pienso sin mayores } \\
\text { problemas*. }\end{array}$} \\
\hline \multicolumn{4}{|l|}{ 5. Generalmente le caigo bien a la gente. } \\
\hline \multicolumn{4}{|l|}{$\begin{array}{l}\text { 6. Siento que podré lograr las metas que me } \\
\text { proponga*. }\end{array}$} \\
\hline \multicolumn{4}{|l|}{$\begin{array}{l}\text { 7. Cuento con personas que me ayudan si lo } \\
\text { necesito. }\end{array}$} \\
\hline \multicolumn{4}{|l|}{$\begin{array}{l}\text { 8. Creo que en general me llevo bien con la } \\
\text { gente. }\end{array}$} \\
\hline \multicolumn{4}{|l|}{$\begin{array}{l}\text { 9. En general hago lo que quiero, soy poco } \\
\text { influenciable*. }\end{array}$} \\
\hline \multicolumn{4}{|l|}{$\begin{array}{l}\text { 10. Soy una persona capaz de pensar en un } \\
\text { proyecto para mi vida. }\end{array}$} \\
\hline \multicolumn{4}{|l|}{$\begin{array}{l}\text { 11. Puedo aceptar mis equivocaciones } y \\
\text { tratar de mejorar. }\end{array}$} \\
\hline \multicolumn{4}{|l|}{$\begin{array}{l}\text { 12. Puedo tomar decisiones sin dudar } \\
\text { mucho. }\end{array}$} \\
\hline $\begin{array}{l}\text { 13. En caro sin mayores problemas mis } \\
\text { obligaciones diarias. }\end{array}$ & & & \\
\hline
\end{tabular}




\section{Apéndice 4}

\section{ESCALA DE CVP - 35}

A continuación, le presentamos una serie de frases acerca de situaciones relacionadas con su trabajo. Piense en su situación laboral, deberá responder dentro de una escala del 1 al 10 , donde 1 es nada y 10 es mucho. Debe marcar con un aspa $(\mathrm{X})$ sobre el recuadro del número que le corresponde, según la siguiente escala:

\begin{tabular}{|c|c|c|c|c|c|c|c|c|c|c|}
\hline \multirow[b]{2}{*}{ Ítems } & \multicolumn{2}{|c|}{ NADA } & \multicolumn{3}{|c|}{ ALGO } & \multicolumn{3}{|c|}{ BASTANTE } & \multicolumn{2}{|c|}{ MUCHO } \\
\hline & 1 & 2 & 3 & 4 & 5 & 6 & 7 & 8 & 9 & 10 \\
\hline 1. Cantidad de trabajo que tengo. & & & & & & & & & & \\
\hline $\begin{array}{l}\text { 2. Prisas y agobios por la falta de tiempo } \\
\text { para realizar mi trabajo. }\end{array}$ & & & & & & & & & & \\
\hline $\begin{array}{l}\text { 3. Presión que recibo para realizar la } \\
\text { cantidad de trabajo que tengo. }\end{array}$ & & & & & & & & & & \\
\hline $\begin{array}{l}\text { 4. Presión que recibo para realizar la } \\
\text { cantidad de trabajo que tengo. }\end{array}$ & & & & & & & & & & \\
\hline $\begin{array}{l}\text { 5. Los conflictos que tengo con otras } \\
\text { personas de mi trabajo. }\end{array}$ & & & & & & & & & & \\
\hline $\begin{array}{l}\text { 6. La falta de tiempo para mi vida } \\
\text { personal. }\end{array}$ & & & & & & & & & & \\
\hline 7. La incomodidad física en el trabajo. & & & & & & & & & & \\
\hline 8. La carga de responsabilidad. & & & & & & & & & & \\
\hline 9. Las interrupciones molestas. & & & & & & & & & & \\
\hline $\begin{array}{l}\text { 10. El estrés que tengo (esfuerzo } \\
\text { emocional). }\end{array}$ & & & & & & & & & & \\
\hline $\begin{array}{l}\text { 11. Mi trabajo tiene consecuencias } \\
\text { negativas para mi salud. }\end{array}$ & & & & & & & & & & \\
\hline 12. Satisfacción con el tipo de trabajo. & & & & & & & & & & \\
\hline $\begin{array}{l}\text { 13. La motivación que experimento } \\
\text { (ganas de esforzarme). }\end{array}$ & & & & & & & & & & \\
\hline $\begin{array}{l}\text { 14. La exigencia de capacitación para } \\
\text { realizar mi trabajo. }\end{array}$ & & & & & & & & & & \\
\hline 15. El apoyo de mi familia. & & & & & & & & & & \\
\hline 16. Mis ganas de ser creativo(a). & & & & & & & & & & \\
\hline $\begin{array}{l}\text { 17. La capacitación que tengo para hacer } \\
\text { mi trabajo actual. }\end{array}$ & & & & & & & & & & \\
\hline $\begin{array}{l}\text { 18. Mi trabajo es importante para la vida } \\
\text { de otras personas. }\end{array}$ & & & & & & & & & & \\
\hline 19. Me siento orgulloso(a) de mi trabajo. & & & & & & & & & & \\
\hline
\end{tabular}




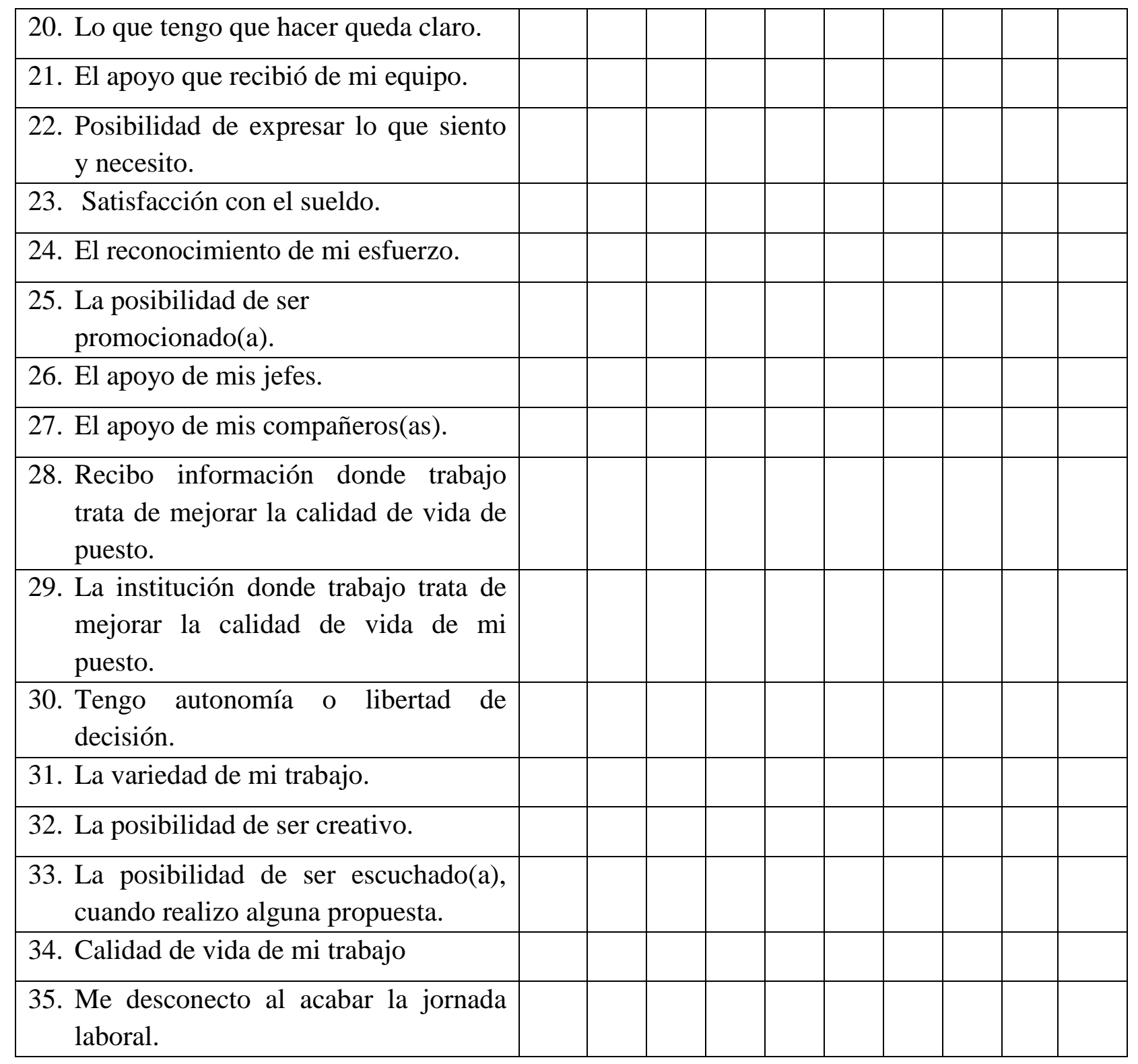

